\title{
Erratum to: Rendezvous based routing protocol for wireless sensor networks with mobile sink
}

\author{
Suraj Sharma ${ }^{1}$ - Deepak Puthal ${ }^{2}$. \\ Sanjay Kumar Jena ${ }^{3}$ - Albert Y. Zomaya ${ }^{4}$. \\ Rajiv Ranjan ${ }^{5,6}$
}

\section{Erratum to: J Supercomput DOI 10.1007/s11227-016-1801-0}

The authors very much regret that errors have slipped into their contribution. The corrections are listed below.

\section{Related works}

The last two paragraphs should read:

An energy-efficient routing protocol called ring routing has been proposed by Tunca et al. [10]. It establishes a ring structure that aims to combine the easy accessibility of the grid structures and the easy changeability of the backbone structure. Since it incorporates a minimal number of nodes in the ring structure, the redundancy of data packets is significantly reduced for sharing sink position advertisement packets among the ring nodes. It devises a straightforward and efficient mechanism. The ring

The online version of the original article can be found under doi:10.1007/s11227-016-1801-0.

$\triangle$ Suraj Sharma

suraj@iiit-bh.ac.in

1 Department of Computer Science and Engineering, IIIT Bhubaneswar, Khurda, India

2 CSIRO Data61, Sydney, Australia

3 Department of CSE, National Institute of Technology Rourkela, Rourkela, India

4 School of Information Technologies, The University of Sydney, Sydney, Australia

5 School of Computing Science, Newcastle University, Newcastle upon Tyne, UK

6 School of Computer Science, China University of Geosciences, Beijing, China 
can be constructed with low overhead unlike the structures utilized in the area-based approaches as in LBDD and Railroad. On the other hand, ring routing relies on the minimum amount of inefficient broadcasts which are extensively used in area-based protocols. The drawback of the above protocols is the mobility management cost and the end-to-end delay.

A vertical and horizontal segregation-based data dissemination protocol for WSNs with mobile sink is proposed by Jain et al. [33]. The proposed method mainly focuses on message broadcasting and is not always efficient for all the applications of sensor networks. As there is no empirical validation provided for the proposed method, its applicability in real-world setting is debatable. In contrast, the focus of our work in this paper is to propose new methods for implementing mobile sink management and efficient data transmissions considering different level of heterogeneities and complexities. To this end, we develop two concrete methods. In the first method, the source node transmits the data to the closest backbone-tree node and backbone-tree node relays the data to the sink. While in the second method, the source node retrieves the sink location from the nearest backbone-tree node and transmits the data directly to the sink using the sink location. Unlike [33], which only considers theoretical analysis of the proposed method, we have to validate the proposed methods by conducting extensive simulation using the Castalia (v3.2) simulator.

\subsection{Tree construction}

The correct version of Algorithm 2 is given below.

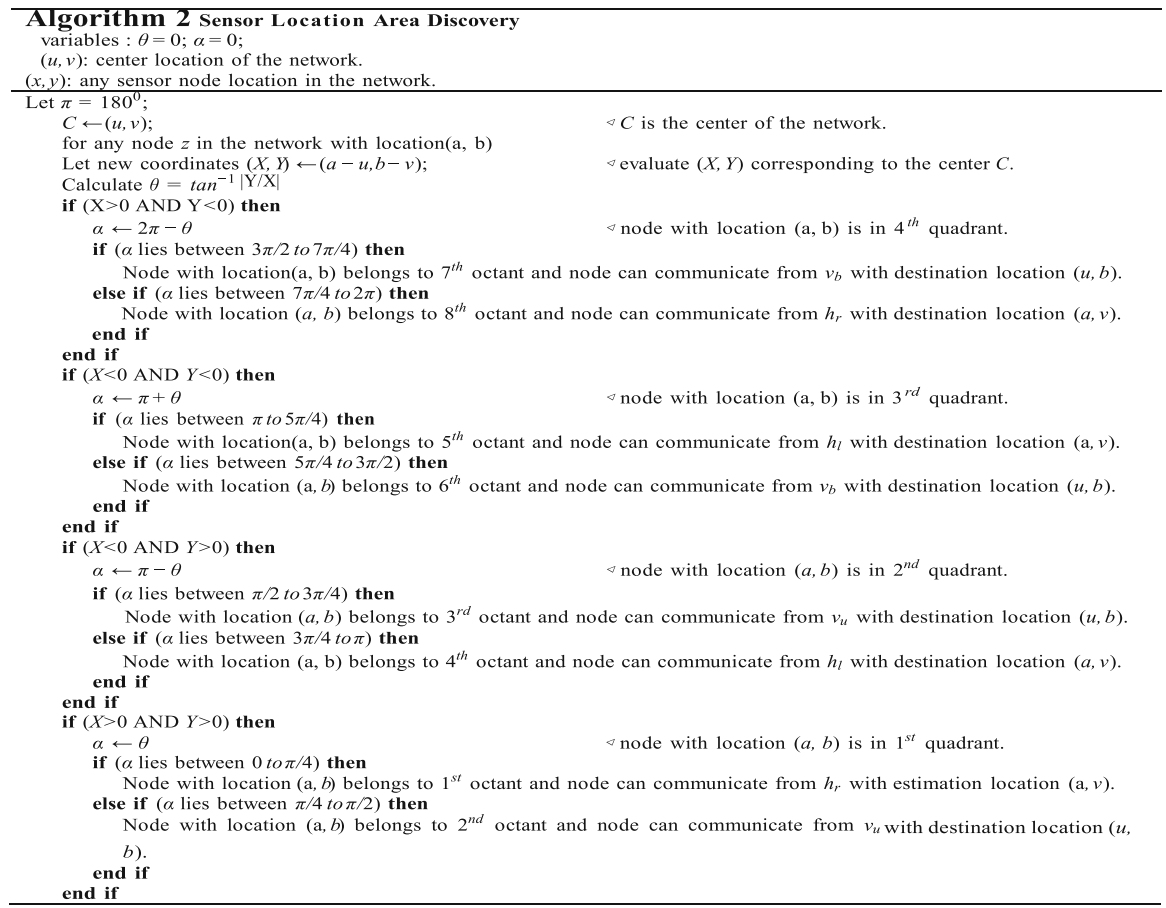




\section{References}

The citation Jain et al. [33] mentioned in the last paragraph of Sect. 2 (Related works) is as follows:

33. Jain S, Sharma S, Bagga N (2016) A vertical and horizontal segregation based data dissemination protocol. Emerging research in computing, information, communication and applications. Springer, India, pp 401-412 